\title{
NON-NEOFORMANS CRYPTOCOCCEMIA IN A PATIENT WITH HODGKIN'S LYMPHOMA
}

\author{
CHUAN HUN DING ${ }^{1 *}$, NORHIDAYAH KAMARUDIN ${ }^{2}$
}

${ }^{1}$ Department of Medical Microbiology and Immunology, Faculty of Medicine, Universiti Kebangsaan Malaysia, Kuala Lumpur, Malaysia. ${ }^{2}$ Department of Pathology and Laboratory Medicine, Kulliyyah of Medicine, International Islamic University Malaysia, Kuantan Campus, Pahang, Malaysia. Email: dingch@ppukm.ukm.edu.my

Received: 19 September 2018, Revised and Accepted: 19 October 2018

ABSTRACT

Cryptococcemia attributed to non-neoformans cryptococci has not received much attention due to the relative scarcity of reported cases. The primary aim of this case report is to highlight several notable characteristics of Cryptococcus laurentii. A 35-year-old woman presented with a right supraclavicular mass and a history of low-grade fever. A yeast was isolated from her blood. It formed cream-colored colonies which turned yellowish after $72 \mathrm{~h}$ of incubation and had a microscopic morphology of elongated budding yeast cells. Its identity was confirmed to be $C$. laurentii through carbohydrate assimilation testing (ID $32 \mathrm{C}$ ). The patient responded clinically to fluconazole and cleared the cryptococcemia. She was subsequently diagnosed with Hodgkin's lymphoma through histopathological examination of the supraclavicular mass.

Keywords: Cryptococcus laurentii, Fluconazole, Hodgkin's lymphoma.

(c) 2018 The Authors. Published by Innovare Academic Sciences Pvt Ltd. This is an open access article under the CC BY license (http://creativecommons. org/licenses/by/4. 0/) DOI: http://dx.doi.org/10.22159/ajpcr.2018.v11i12.29833

\section{INTRODUCTION}

Non-neoformans cryptococci are generally regarded as non-pathogenic saprophytes [1,2]. Moreover, it has also been established that Cryptococcus laurentii (a non-neoformans Cryptococcus species) is less virulent than Cryptococcus neoformans [3]. However, as the number of immunocompromised patients increases, so must the recognition that non-neoformans cryptococci can become clinically important human pathogens [4]. Although non-neoformans cryptococci can also cause serious infections (e.g., central nervous system and bloodstream infections), the clinical manifestations are often indistinguishable from those attributable to C. neoformans [5]. C. laurentii has been reported to have reduced susceptibility to fluconazole [5], a commonly prescribed antifungal agent which may be employed to treat $C$. neoformans infections. This case report highlights some notable characteristics of $C$. laurentii which may be important for diagnostic and clinical purposes.

\section{CASE REPORT}

A 35-year-old woman presented to a tertiary medical center for investigation of a right supraclavicular mass. The mass was initially a painless and thumb-sized swelling when it was first noticed by the patient 5 months back. Subsequently, the mass progressively increased in size and became painful. The local symptoms were also associated with low-grade fever. On examination, the patient was fully conscious, not tachypneic but febrile with a body temperature of $37.6^{\circ} \mathrm{C}$. A large swelling was noted at her right supraclavicular region, measuring $20 \times 15 \mathrm{~cm}$. A sinus was seen to discharge hemoserous fluid. The mass was firm and fixed to the overlying skin. No other neck masses or lymph nodes were palpable. Examination of respiratory, cardiovascular, and other systems was unremarkable. A provisional diagnosis of staphylococcal supraclavicular abscess was made and she was empirically treated with intravenous cloxacillin $500 \mathrm{mg}$ q6h.

Preliminary laboratory investigations of the patient's blood revealed leukocytosis (total white cell count of $21.8 \times 10^{9}$ cells $/ \mathrm{L}$ ) with evidence of neutrophilia $\left(18.1 \times 10^{9}\right.$ cells $\left./ \mathrm{L}\right)$ and a raised C-reactive protein level ( $9.22 \mathrm{mg} / \mathrm{dL})$. Her retroviral screening was non-reactive. A swab of the discharge and a blood specimen were sent for microbiological culture.
The supraclavicular mass was also biopsied for histopathological examination. After 4 days of incubation, the blood specimen in the BACTEC Myco/F Lytic vial was positive for microbial growth. A Gram stain revealed elongated and budding yeast cells with no pseudohyphae. The yeast was cultured on Sabouraud dextrose agar (SDA) and cream-colored colonies were observed after $48 \mathrm{~h}$ of incubation at $35^{\circ} \mathrm{C}$, which turned yellowish after $72 \mathrm{~h}$. On cornmeal agar, elongated and budding yeast cells were observed under the light microscope (Fig. 1). Through carbohydrate assimilation tests (ID 32 C; bioMerieux, France), the yeast was finally identified as C. laurentii (numerical profile: 5777377371, 99.0\% identification). The swab specimen grew coagulase-negative staphylococci and the bacteria were regarded as skin contaminants.

Following the release of the blood culture report, intravenous cloxacillin was discontinued after 6 days and a course of intravenous fluconazole $400 \mathrm{mg}$ OD was started. A follow-up blood culture after a week of fluconazole treatment was negative for both bacteria and fungi. The patient responded clinically to the antifungal therapy and was allowed home after completing a 2-week course of fluconazole. Two weeks post-admission, the histopathological report for the supraclavicular mass (lymph node) provided a diagnosis of classical Hodgkin's lymphoma. Fungal stains (i.e., periodic acid-Schiff and Grocott-Gomori methenamine silver) did not reveal the presence of any fungal elements/bodies in the tissue specimen.

\section{DISCUSSION}

Non-neoformans cryptococci are encapsulated yeasts with a worldwide prevalence and they can be isolated from numerous environmental sources and foods [5]. The term "non-neoformans cryptococci" traditionally refers to Cryptococcus spp. other than C. neoformans and Cryptococcus gattii because the latter was previously considered a variant of $C$. neoformans [4]. Approximately $80 \%$ of non-neoformans cryptococcal infections are caused by C. laurentii and Cryptococcus albidus [4]. Most cases of nonneoformans cryptococcal fungemia are nosocomially acquired and the presence of invasive devices is a significant predisposing factor particularly when associated with C. laurentii infections [6]. Although in the present case, the patient did not have any indwelling medical device (e.g., central venous line), by having a hematological 


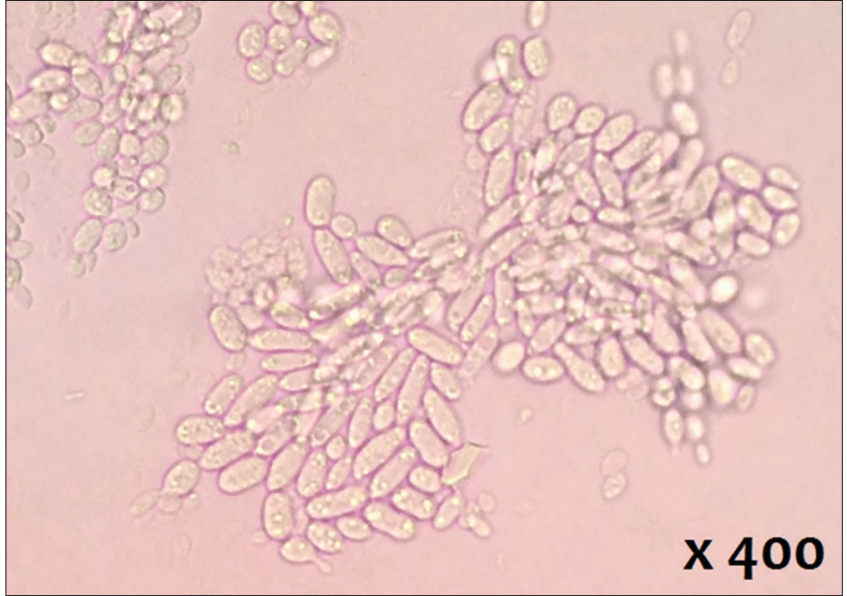

Fig. 1: Microscopic morphology of Cryptococcus laurentii cultured on cornmeal agar

malignancy (i.e., lymphoma), her cell-mediated immunity could have been compromised, leading to the $C$. laurentii cryptococcemia [4]. Although $C$. laurentii fungemia has been reported before in patients with underlying non-Hodgkin's lymphoma [4,7], to the best of our knowledge, there has been no report associating the infection with classical Hodgkin's disease.

Although C. laurentii is India Ink positive and produces urease, these characteristics are common to all cryptococci. However, the capsule of C. laurentii in the India Ink mount is narrow/slight [1,8]. Furthermore, its microscopic morphology is that of elongated and budding yeast cells [9] rather than the globose yeast cells which are more typical of $C$. neoformans and C. gattii. Another clue to the presence of $C$. laurentii is yellow (or orange) colonies on SDA, especially after extended incubation [10]. A negative caffeic acid test is another supplementary test which would distinguish $C$. laurentii from $C$. neoformans [8]. Non-neoformans cryptococci are less likely to produce positive results in cryptococcal antigen detection tests [4], and thus, glucuronoxylomannan antigen assays should not be relied on to diagnose cases of $C$. laurentii fungemia. For laboratories without molecular identification (i.e., DNA sequencing) capabilities, commercial carbohydrate assimilation kits for yeasts such as ID $32 \mathrm{C}$ (bioMerieux, France) may be utilized [8].

There are no established guidelines for the treatment of infections caused by $C$. laurentii $[9,10]$. However, with regard to the in vitro susceptibility profile of $C$. laurentii, a study on 39 isolates revealed that amphotericin $B$ was the most active compound, fluconazole had limited activity, and flucytosine as well as echinocandins were inactive [2]. In contrast, fluconazole resistance has been virtually absent in C. neoformans isolates from Asia [11]. Admittedly, we did not perform antifungal susceptibility testing on our $C$. laurentii isolate because there were no published Clinical and Laboratory Standards Institute breakpoints for the antifungal susceptibility testing of cryptococci. We were, however, fortunate that our patient responded clinically to fluconazole. Other authors have also reported positive outcomes with fluconazole therapy, either as a sole therapeutic agent [9] or as a subsequent antifungal agent following amphotericin B therapy [1]. Other azoles (e.g., voriconazole) were also used successfully to treat infections caused by C. laurentii [10]. The recent discovery of new antifungal compounds from plants such as fenugreek and clove basil could also potentially expand the antifungal arsenal against $C$. laurentii $[12,13]$.

\section{CONCLUSION}

All clinical microbiology laboratories should attempt to identify non-neoformans cryptococci to the species level to aid therapeutic decisions. Due to the unpredictable fluconazole susceptibility profile, the threshold to switch to amphotericin B should be low for patients with $C$. laurentii infections who are initially managed with fluconazole.

\section{ACKNOWLEDGMENT}

We would like to thank the Dean of the Faculty of Medicine, Universiti Kebangsaan Malaysia, for her motivation and permission to publish this article. We are also grateful for the technical assistance provided by Prof. Dr. Srijit Das from the Department of Anatomy, Faculty of Medicine, Universiti Kebangsaan Malaysia.

\section{AUTHOR'S CONTRIBUTIONS}

Study conception and design: Chuan Hun Ding. Collection and data analysis: Norhidayah Kamarudin. Drafting of article and revising it critically for important intellectual content: Chuan Hun Ding and Norhidayah Kamarudin. Final approval of the version to be submitted: Chuan Hun Ding and Norhidayah Kamarudin.

\section{CONFLICTS OF INTEREST}

The authors declare that they have no conflicts of interest.

\section{REFERENCES}

1. Banerjee P, Haider M, Trehan V, Mishra B, Thakur A, Dogra V, et al. Cryptococcus laurentii fungemia. Indian J Med Microbiol 2013;31:75-7.

2. Bernal-Martinez L, Gomez-Lopez A, Castelli MV, Mesa-Arango AC, Zaragoza O, Rodriguez-Tudela JL, et al. Susceptibility profile of clinical isolates of non-Cryptococcus neoformans/non-Cryptococcus gattii Cryptococcus species and literature review. Med Mycol 2010;48:90-6.

3. Andrade-Silva L, Ferreira-Paim K, Silva-Vergara ML, Pedrosa AL. Molecular characterization and evaluation of virulence factors of Cryptococcus laurentii and Cryptococcus neoformans strains isolated from external hospital areas. Fungal Biol 2010;114:438-45.

4. Khawcharoenporn T, Apisarnthanarak A, Mundy LM. Non-neoformans cryptococcal infections: A systematic review. Infection 2007;35:51-8.

5. Miceli MH, Díaz JA, Lee SA. Emerging opportunistic yeast infections. Lancet Infect Dis 2011;11:142-51.

6. Ajesh K, Sreejith K. Cryptococcus laurentii biofilms: Structure, development and antifungal drug resistance. Mycopathologia 2012;174:409-19.

7. Kremery V, Krupova I, Denning DW. Invasive yeast infections other than Candida spp. in acute leukaemia. J Hosp Infect 1999;41:181-94.

8. Cheng MF, Chiou CC, Liu YC, Wang HZ, Hsieh KS. Cryptococcus laurentii fungemia in a premature neonate. J Clin Microbiol 2001;39:1608-11.

9. Shankar EM, Kumarasamy N, Bella D, Renuka S, Kownhar H, Suniti S, et al. Pneumonia and pleural effusion due to Cryptococcus laurentii in a clinically proven case of AIDS. Can Respir J 2006;13:275-8.

10. Bhat V, Vira H, Khattry N, Toshniwal M. Cryptococcus laurentii diarrhea post hematopoietic stem cell transplant. Transpl Infect Dis 2017; 19:e12663.

11. Pfaller MA, Messer SA, Jones RN, Castanheira M. Antifungal susceptibilities of Candida, Cryptococcus neoformans and Aspergillus fumigatus from the Asia and Western Pacific region: Data from the SENTRY antifungal surveillance program (2010-2012). J Antibiot 2015;68:556-61

12. Dharajiya D, Jasani H, Khatrani T, Kapuria M, Pachchigar K, Patel P. Evaluation of antibacterial and antifungal activity of fenugreek (Trigonella foenum-graecum) extracts. Int $\mathrm{J}$ Pharm Pharm Sci 2016;8:212-7.

13. Pandey S. Antibacterial and antifungal activities of Ocimum gratissimum L. Int J Pharm Pharm Sci 2017;9:26-31. 